\title{
30 BLOOD-BASED GLYCOPROTEIN SIGNATURES IN ADVANCED NON-SMALL-CELL LUNG CARCINOMA (NSCLC) RECEIVING FIRST-LINE IMMUNE CHECKPOINT BLOCKADE
}

${ }^{1}$ Klaus Lindpaintner*, ${ }^{2}$ Michael Cheng, ${ }^{3}$ jillian Prendergast, ${ }^{3}$ Karl Normington, ${ }^{1}$ Maurice Wong, 'Gege Xu, ${ }^{1}$ Xini Cong, ${ }^{1}$ Rachel Rice, ${ }^{2}$ Marissa Lawrence, ${ }^{2}$ Kesi Michael, ${ }^{1}$ Daniel Serie. ${ }^{1}$ InterVenn, Concord, NH, USA; ${ }^{2} D F C I$, Boston, MA, USA; ${ }^{3}$ Palleon, Waltham, MA, USA

Background Immune checkpoint blockade is an integral component of first-line therapy for most patients with ad-vanced non-small cell lung cancer (NSCLC), however individual patient outcomes are highly variable and improved biomarkers are needed. Protein glycosylation is an emerging mechanism of immune evasion in cancer. We examined blood-based glycopeptide signatures in a cohort of advanced NSCLC patients treated with first-line immune checkpoint blockade.

Methods Pretreatment blood samples were obtained from 46 advanced NSCLC patients treated with first line pembrolizumab or pembrolizumab + carboplatin + pemetrexed. All patients provided written in-formed consent to the institutional review board-approved protocols (\#02-180 and 13367 ) at the Da-na-Farber/Harvard Cancer Center (Boston, MA), and the study was conducted in accordance with the Declaration of Helsinki. Samples were analyzed using an advanced glycoproteomics platform (Inter-Venn Biosciences) that combines ultra-high-performance liquid chromatography coupled to triple quadrupole mass spectrometry with a proprietary neural-network-based data processing engine. 409 individual glycopeptide (GP) signatures derived from 67 abundant serum proteins were analyzed and correlated with overall survival (OS) and other clinical outcomes.

Results We identified 30 GPs with abundance differences using a False Discovery Rate (FDR) threshold of 0.05 . Using the 5 most predictive GP markers, we created a multivariable model for OS by generating leave-one-out cross-validation (LOOCV) scores and determining an optimized cutoff value of -0.83 (range: -2.2 - 3.4) for these scores using Harrell's concordance index. The median overall survival was 2.8 years for patients $(n=14)$ whose GP classifier value was above the cutoff and 0.8 years for patients $(n=32)$ whose GP classifier value was below the cutoff (HR 7.4, 95\% CI 1.7-32.1, $\mathrm{p}=0.007$ ) The model's perfor-mance was not affected by sex, age, or treatment regimen.

Conclusions Blood-based glycopeptide signatures may represent novel, non-invasive biomarkers of clinical out-come to firstline immune checkpoint blockade in advanced NSCLC. Additional research is needed to validate these findings in larger cohorts and to explore potential applications relevant to clinical decision-making.

Ethics Approval The study obtained ethics approval from the institutional review board (approved protocol \#02-180 and 13-367) at the Dana-Farber/Harvard Cancer Center (Boston, $\mathrm{MA}$ ), and the study was conducted in accordance with the Declaration of Helsinki.

Consent All patients provided written informed consent to the institutional review board-approved protocols (\#02-180 and 13-367) at the Dana-Farber/Harvard Cancer Center (Boston, MA), and the study was conducted in accordance with the Declaration of Helsinki.

http://dx.doi.org/10.1136/jitc-2021-SITC2021.030 